\title{
ERZÄHLWENDE IN HANDKES DER KURZE BRIEF ZUM LANGEN ABSCHIED
}

Anđelka Krstanović, Univerzität Banja Luka, kandelka@gmail.com, andjelka.krstanovic-jankovic@flf.unibl.org

Original scientific paper

DOI: $10.31902 /$ fll.33.2020.6

UDC: 821.112.2 (436).09-31

\begin{abstract}
Peter Handkes Erzählung Der kurze Brief zum langen Abschied stellt neben Wunschloses Unglück, ebenso 1971 entstanden, eine entscheidende Wende in Bezug auf narrative Strategien dar. Während Handke in den 1960erJahren mit traditionellen Erzählverfahren experimentiert, sublimiert er Anfang 1970er-Jahren diese Erfahrungen und vermittelt authentische Geschichten mit neugewonnen Erzählstrategien. Die angewandten Verfahren in den oben genannten Werken stellen somit eine Wende im Opus des Autors dar, die Handke als Grundkonstituenten seiner Poetik auch in späteren Werken beibehalten hat.

In dem vorliegenden Beitrag werden Erzählverfahren in Der kurze Brief zum langen Abschied analysiert, um die genannte Wende näher zu verdeutlichen. Zudem wird die Position dieser Erzählung im Gesamtwerk des Autors interpretiert.
\end{abstract}

Schlüsselwörter: Peter Handke, Erzählung, Erzählstrategien, Erzählwende, Poetik.

\section{Einleitung}

Der kurze Brief zum langen Abschied hat als biografischen Hintergrund Handkes Reise nach Amerika, die Eheprobleme und ein komplexes Verhältnis zur Kindheit und der Familie. Jeder dieser biografischen Punkte gehört zur Geschichte. Aus diesem Grund wird die Erzählung dem Neuen Subjektivismus zugeordnet (vgl. Brüggemann, 1996), eine Tendenz in der Literaturentwicklung der 1970er-Jahre, die durch Verlegung der privaten Geschichten in den fiktionalen Rahmen gekennzeichnet war. Es zeigte sich aber, dass dieses Verhältnis des Autors zur Geschichte komplexere Ansprüche an die eigene Poetik stellt und die Grundtendenz des Neuen Subjektivismus - den Drang nach Bekenntnis - bei Weitem übersteigt. ${ }^{1}$

${ }^{1}$ Des Autors Spiel mit dem Biografischen übersteigt die Tendenzen des Neuen Subjektivismus. Im Kontext dieses Anspruchs ist der Beitrag von Edit Király „On the 


\section{Handlung, Figuren, Raum}

Die Handlung in Der kurze Brief zum langen Abschied erstreckt sich auf die Tage, die ein autodiegetischer Erzähler in Amerika verbringt. Mit genügend Geld ausgestattet, unternimmt er eine Rundreise quer durch Amerika. Seine Stationen sind Providence, New York, Philadelphia, St. Louis, Estacada und Kalifornien. Bei der Ankunft in Providence bekommt er einen seltsamen Brief von seiner Ehefrau Judith, die inn verlassen hat: „Ich bin in New York. Bitte such mich nicht, es wäre nicht schön, mich zu finden." (Der kurze Brief zum langen Abschied 2001, 11) Nach dem Inhalt des Briefes ist der erste Teil der Erzählung mit Der kurze Brief betitelt. Der Erzähler lässt sich von seiner Ehefrau verfolgen, wird von ihr ständig bedroht, überfallen und beinahe ermordet, um sich schließlich mit ihr zu versöhnen und einen gemeinsamen Besuch im Garten des Regisseurs John Ford zu genießen. Das ist die Oberflächenstruktur der Geschichte, die einerseits durch die lineare Abfolge ihrem Schluss zustrebt, andererseits durch Zustandsbeschreibungen und Selbstanalysen diese Kontinuität unterbricht. Die Schilderungen in Form von lang gedehnten Sequenzen verleihen der Geschichte Merkmale einer statischen Struktur. Daraus ergibt sich die Tiefenstruktur der Geschichte, die, abstrakt formuliert, die Suche nach einer Bewusstseinsveränderung thematisiert.

Der autodiegetische Erzähler kommt nach Amerika, um seine Eheprobleme zu lösen, aber auch um andere Bindungen und Lebensformen $^{2}$ aus der Vergangenheit zu hinterfragen, die einen offenen Bezug zur Welt verhinderten. Die Eheprobleme mit Judith sind nur die Auslöser einer tieferen Identitätskrise. Die Voraussetzungen für die Bewältigung dieser Krise werden vom Erzähler initiiert. Er unternimmt eine Reise, um in Distanz zu treten. Auf dieser Reise wird er mit anderen Lebensformen konfrontiert, um Vergleiche ziehen zu können. Ferner begegnet er neuen Menschen, die ihm einen neuen Erfahrungshorizont aufzeigen, und zugleich liest er viel. Die Bildung als weiteres Motiv hilft ihm, seine Lebenssituation besser zu verstehen und zu neuen Möglichkeiten der Veränderung zu gelangen. Die genannten Motive stehen für die Gegenwart und stellen über das Motiv der Verfolgung eine Brücke zur Vergangenheit her. Judith

road with Peter Handke. Amerikanische (T)räume in der Erzählung Der kurze Brief zum langen Abschied" aufschlussreich. In: Das Wort sei gewagt. (Hg): Attila Bombitz und Katharina Pektor. Wien: Praesens, 2019, 89.

2 Die im weiteren Text variierenden Ausdrücke wie Lebenskonzept, Lebensart, Lebensweise, laufen auf diesen von Handke konstruierten Begriff Lebensform hinaus, der die Stellung des Subjekts zu seinem Dasein und die Behauptung seiner existenziellen Position in dem dargegebenen Umfeld umfasst. 
verfolgt den Erzähler, und diese Verfolgung spiegelt alle weiteren Verfolgungen aus der Vergangenheit wider, die in Form von Ängsten und panischen Anfällen in das Bewusstsein des Erzählers eindringen. Für die Vergangenheit stehen die Motive der Ehe und der Kindheit. Beide sind negativ konnotiert, der Erzähler leidet darunter und möchte sie überwinden. Der Schnittpunkt zwischen Gegenwart und Vergangenheit ist die Erinnerung, die nicht auf bloßes Wiederholen von Erlebnissen zurückgeführt, sondern in den gegenwärtigen Prozess der qualitativen Veränderung des Bewusstseins umgesetzt wird. Das Motiv der Erinnerung füllt die Zustandsbeschreibungen und Sequenzen mit Selbstanalysen. Es hat die Funktion, in einer neuen Umgebung die alten Leiden ins Bewusstsein zu rufen, sie zu formulieren und vor dem Hintergrund der neuen Erfahrungen in einen produktiven Bewusstseinsvorgang zu überführen. Diese Absicht, durch eine tätige Erinnerung Ansätze von Hoffnung für das Bewusstsein zu gewinnen, äußert der Erzähler im Gespräch mit Claire (vgl. Der kurze Brief 82).

Alle Motive münden in das Thema der Bewusstseinsveränderung, eine Aufgabe, die sich der Erzähler auf der Reise durch Amerika stellt. Um dieses Ziel zu erreichen, muss er einen mühevollen Prozess der Trennung von alten Lebensformen durchlaufen und einen neuen konfliktfreien Zugang zum sozialen Umfeld finden. Dieser Vorgang wird durch Zustandsbeschreibungen veranschaulicht, lange selbstanalytische Phasen, nach denen die Lösungen als plötzliche Geschehnisse im Bewusstsein des Erzählers sich ruckartig abzeichnen. Die qualitativen Veränderungen des Bewusstseins treiben die Tiefenstruktur der Geschichte voran.

Als Figuren sind Judith, Claire, Benedictine und John Ford mit Eigennamen verzeichnet. Die hervorgehobene Stellung deutet darauf hin, dass sie den Kern um die Geschichte der Veränderung bilden. Sie verursachen, begleiten, determinieren und bestätigen die innere Veränderung des Erzählers. Die übrigen Figuren fungieren als Rollenträger, so die Mutter, der Bruder, der Soldat, der Sänger, das Kind und das Liebespaar, und sie kreisen satellitenhaft um das Thema Bewusstseinsveränderung, indem sie das Gerüst der Handlung auf einer erweiterten oppositionellen Achse zusätzlich ergänzen. Die Figuren stehen für die Vertreter der zwei verschiedenen Zeichensysteme, das alte, das Judith, die Mutter und der Bruder repräsentieren, und das neue amerikanische Zeichensystem, das wiederum Claire, das Kind, das Liebespaar und John Ford vertreten. Daraus ergibt sich eine oppositionelle Aufteilung der Figuren, die zugleich Einfluss auf die Entwicklung des Erzählers ausüben. Die Begegnungen mit Menschen in Amerika, die eine neue Lebensform 
verkörpern, ermöglichen dem Erzähler, Vergleiche zu seinen früheren Beziehungen zu ziehen und eine neue Wahrnehmungsweise zu entwickeln. Die Konflikte werden im Inneren des Erzählers zwischen dem Erzähler und den Vertretern der Vergangenheit ausgetragen. Deutlich werden diese Konflikte durch zwei verschiedene Liebeserfahrungen. Die oppositionelle Konstellation der weiblichen Figuren Judith und Claire markiert die Kluft zwischen zwei Welten. Während die eheliche Bindung mit Judith, die vom Rollenhaften und einer zwanghaften Abhängigkeit gesteuert war, alles Spontane und Persönliche infrage stellte, bezeichnet die Bindung mit Claire ein auf freiem Wechselbezug basierendes Verhältnis. Die Beziehung, spontan zu leben, anstatt diese vorgeschrieben zu betreiben, ist durch Claire eine neue Erfahrung geworden, aus welcher der Erzähler Vergleiche zieht und lernt.

Die Ehe mit Judith ist gescheitert, die Ehepartner müssen aber noch einen schmerzlichen Trennungsprozess durchlaufen. Dieser ist durch Angst, Panik, Hass und schließlich Versöhnung gekennzeichnet. Während Judith in ihrer Verzweiflung den Erzähler verfolgt, wird deutlich, dass auch der Erzähler sie aufsucht und sich auch seinerseits von ihr noch nicht lösen kann. Gegenüber Judith verspürt er Angst und Wut zugleich. Diese Gefühle, die durch Selbstgespräche thematisiert werden, werden oft zu Projektionen der Ängste aus der Kindheit. So stellt Judith im Innern des Erzählers ein Echo der alten Verhaltensformen dar:

'Ob sie inzwischen tot ist?' Auf einem hohen Felskegel hatte ich einmal gegen Abend nach meiner Mutter gesucht. Sie wurde ab und zu schwermütig, und ich glaubte, sie hätte sich, wenn nicht hinuntergestürzt, so doch einfach hinabfallen lassen [...] (Der kurze Brief 15)

Die Sorge um das Leben von Judith geht in die Erinnerung an die Mutter über. Ebenso an einer weiteren Stelle:

[D]enn als ich sie zuletzt gesehen hatte, an einem Nachmittag langausgestreckt auf ihrem Bett, war sie schon nicht mehr ansprechbar gewesen und hatte so zu mir hergeschaut, daß ich auf dem Weg zu ihr stehenblieb, weil ich ihr nicht mehr helfen konnte. (Der kurze Brief 18) Auch wenn der Erzähler glaubt, ein anderer geworden zu sein, wiederholt sich die Sorge um Judith immer wieder ruckartig durch plötzliche Ausbrüche der Angst (vgl. Der kurze Brief 22, 33). So lässt Judith die Schrecken aus der Kindheit wieder auferstehen, die die Verhaltensformen des Erzählers steuern. Die alten Ängste wiederholen sich in der Beziehung mit Judith, und der Erzähler fühlt sich machtlos 
gegenüber den alten Schrecken, was ihn in Wut versetzt (vgl. Der kurze Brief 57).

Die Entfremdung von der Ehefrau durchläuft die Stationen von Beurteilung, Angst, Panik, Hass und Wut. Der Höhepunkt des Unvermögens, sich vom Paradigma der alten Verhaltensmuster zu lösen, ist die Wut über Judith, welche die Kindheitserlebnisse nur wiederholen lässt: „’Du Ding!' sagte ich. 'Ich schlage dich zu Brei, ich schlage dich zu Brei, ich schlage dich zu Brei. Bitte laß dich nicht finden, du Unwesen. Es wäre nicht schön für dich, von mir gefunden zu werden.'" (Der kurze Brief 61)

Die anfängliche Neigung schrumpfte in der Ehe auf einen rollengesteuerten Umgang miteinander. Dieser ist auf die alte Lebensform zurückzuführen, die der Erzähler von Kindheit an kennt. Er fühlt sich gefangen in einer solchen Lebensform, welche statt des spontanen einen vorgespielten Umgang auferlegt und jede Art der Hoffnung, aus diesem Rahmen aussteigen zu können, als bloßen Traum abstempelt. Er erinnert sich an diese Vorfälle aus der Kindheit:

'Da wir arm waren, erlebte ich fast nur Leute, die auch arm waren. Da wir so wenig Dinge sahen, gab es nicht viel zu reden, und so redeten wir fast jeden Tag das gleiche. Wer mehr redete, war in diesen Verhältnissen, wenn er dabei lustig war und die anderen unterhielt, ein Original, wenn er aber nur schwärmte wie ich, ein Träumer [...]. Und diese Träume waren in der Umwelt, in der ich lebte, wirklich Schwärmereien, weil es für sie in dieser Umwelt keine Entsprechung gab, nichts Vergleichbares, das sie möglich gemacht hätte.' (Der kurze Brief 80)

Mit Judith erlebt er erneut diesen Kreis der Enge und des Ausgeschöpften. Auf der Reise nach Amerika wird er aber mit neuen Wahrnehmungsbildern vertraut, die keine Schwärmereien sind, sondern reale Bilder einer anderen Welt, was ihm ermöglicht, Vergleiche zu ziehen. Das ist ein neues qualitatives Moment, das in der Kindheit nicht vorstellbar war, und das den Traum eines Weges zu einer persönlichen Entwicklung möglich macht.

Der Erzähler beschreibt Judith als eine Person, die ihr Nichtzurechtfinden in der Welt hinter verschiedenen Rollenmasken versteckt. Nur auf der Bühne sah er sie im Gleichgewicht mit sich selbst (vgl. Der kurze Brief 48). Judith wird selbstlos nur im Rahmen einer bestimmten Rolle, sie verkörpert dadurch eine Lebensform, die der Erzähler einerseits als eine vorgespielte erlebt und die andererseits seine persönliche Entwicklung zwanghaft determiniert hat. Sie hat ihm die Möglichkeit einer spontanen Beziehung auf eine lange Zeit versperrt (vgl. Der kurze Brief 36). 
Da der Erzähler von Kindheit an seine eigenen Wünsche, Neigungen und Gefühle als unerwünschte Träume erleben musste, ist er nicht in der Lage, einen persönlichen Bezug zur Welt herzustellen. Er bewegt sich immer wieder im Rahmen einer auferlegten Lebensform. Erst durch das Verhältnis mit Claire wird er einen ganzen Überblick über die Beziehung mit Judith gewinnen können und imstande sein, das Verhältnis zu Judith zu beschreiben. Er erzählt Claire die ganze Geschichte mit Judith. Durch die Formulierung erlebt er einen Fortschritt in seinem Bewusstsein, der den Trennungsprozess vorantreibt. Eine endgültige Lösung von der Vergangenheit ist ihm aber zu diesem Zeitpunkt noch nicht möglich (vgl. Der kurze Brief 151). Um diese Grenze zu überschreiten, braucht es noch eine letzte Begegnung mit Judith, die die Verkörperung aller alten Ordnungen darstellt. Bei der letzten Begegnung werden die Rollen gewechselt. Nicht der Erzähler erscheint ohnmächtig, sondern Judith, die mit ihrem Vorhaben, den Ehemann zu erschießen, scheitert (vgl. Winkelmann 143).

Tief enttäuscht von der falschen und ohnmächtigen Vergangenheit, die inn eine so lange Zeit gehemmt hat, lässt er die alten Ordnungen, in Judith verkörpert, vor seinen Augen von selbst auseinanderfallen. Die Begegnung zwischen den beiden und der nachfolgende Besuch bei John Ford markieren zudem, wie es die Analyse von Theo Elm zeigt, eine Lösung aus Entfremdung durch Aufgabe des Besitzdenkens und eine voraussetzungslose Annahme der gegebenen Wirklichkeit (vgl. 69). Damit ist der Prozess der Lösung im Bewusstsein des Erzählers abgeschlossen und sie sind beide bereit, im Garten von John Ford ihre Geschichte hinter sich zu lassen.

Bei der Trennungsgeschichte ist die wesentliche Rolle der oppositionellen weiblichen Figur Claire zuzuschreiben. Durch Claire wird der Erzähler mit der neuen Umgebung vertrauter und kann sich von alten Bindungen allmählich distanzieren (vgl. Neis 52). Claire spielt bei dem Trennungsprozess eine vielfache Rolle. Sie wird zu einem vertrauten Gesprächspartner und aufmerksamen Beobachter der inneren Wandlungen des Erzählers. Zugleich korrigiert sie das Verhaltensmuster des Erzählers. Als Vertreterin der amerikanischen Lebensweise überführt sie den Erzähler in ein neues Lebenskonzept und kommentiert zugleich seine Umstände. Da sie in Bezug auf die Reflexionen über ihr eigenes Leben völlig anspruchslos bleibt, erweist sie sich als ideale Begleiterin für den selbstbezogenen Erzähler. Kurz nach seiner Ankunft nimmt sie alle Veränderungen an ihm präzise wahr und erkennt anhand seiner Redeweise den Drang nach Veränderung (vgl. Der kurze Brief 72, 73). 
Sein Äußeres verweist auf die Wandlung. Er zeigt nicht mehr das Bedürfnis, sich aufzuspielen, und ist lässiger geworden. Die Vergleiche zwischen "früher" und „jetzt" bestätigen, dass er sich von früheren Lebensmustern entfernt und diesen Lösungsprozess auch bewusst in Gang setzt. So beschreibt Claire die innere Veränderung und lässt auch den Erzähler die neugewonnenen Einsichten formulieren. Dies erfolgt in langen Gesprächen, die sie miteinander führen. Außerdem bietet sie ihm einen spontanen Alltag, indem das unmittelbar Menschliche den Rahmen füllt. Dadurch entspannt sich der Erzähler. Claire stellt einen Gegenpol zu ihm dar, denn sie sagt, dass sie sich nicht mehr erinnert. Sie lebt spontan, ohne reflexive Phasen, und repräsentiert so die amerikanische Lebensform des unkonventionellen Handelns. Im Gegensatz zu ihr vertieft der Erzähler immer wieder seine Reflexionen, indem er die Angstzustände, an die er sich erinnert, in Hoffnungsmomente für die Gegenwart umsetzen möchte. Außerdem verbleibt Claire innerhalb eines Zeichensystems und versucht, in diesem vor sich hin zu leben, während der Erzähler den Vorteil nutzt, zwischen zwei Lebensentwürfen vergleichen zu können, um sein das ihm gemäße Konzept herauszufinden (vgl. Der kurze Brief 85).

Die Aussagen von Claire zeigen die Kontraste zwischen Europa und Amerika auf, die zugleich die Schwankungen im Inneren des Erzählers widerspiegeln. Sie formuliert die amerikanische Lebensform, die vom Kollektiv her gesteuert wird, so steht das „Wir" für „Ich“. Das Verhältnis zur Ursprungsgeschichte hat ein zum Mythos erhobenes kollektives Bewusstsein erschaffen, das Einsatz, Tat und eine unmittelbare Gestaltung des Lebens propagiert, was das Lebenskonzept jedes Einzelnen ausmachen soll. So wird die Gegenwart von einer positiv beurteilten Vergangenheit determiniert, während der Erzähler, auf seinen Individualismus bestehend, das persönliche Lebenskonzept in der Loslösung von der Vergangenheit sucht. Claire macht dem Erzähler dieses anders gestaltete Lebenskonzept bewusst und lässt inn durch ihre Perspektive das eigene Bild der Welt korrigieren (vgl. Der kurze Brief 102, 103).

Durch eine therapeutisch wirkende Beobachtung vermittelt Claire dem Erzähler die amerikanische Lebensform, in der die Tat als Veräußerlichung des Lebens wichtiger ist als die Reflexion. So wird der Erzähler mit einem unterschiedlichen Lebenskonzept vertraut, das ihm neue Ausblicke auf die Verwirklichung der eigenen Träume eröffnet. Die gleiche Funktion hat das Liebespaar. Ihre Beziehung kennzeichnet nicht Passivität, sondern ein dynamisches Wechselspiel von kreativen Tätigkeiten. Sie stellen durch eine hermetische Lebensart die 
Zuspitzung der Lebensvorstellungen von Claire dar. Jeder Augenblick ist durch eine konkrete Tat geprägt und das Reflexive ist ihnen fremd. Der Erzähler genießt in dieser Gesellschaft ein allgemeines Lebensgefühl und bedauert, einst von bestimmten Lebensformen gegängelt worden zu sein (vgl. Der kurze Brief 129). Auch die Kunst ist vom praktischen Sinn für das Leben geprägt. Der Maler malt ausschließlich historische Bilder, authentische Geschichten, die das Heldenhafte des amerikanischen Kollektivs widerspiegeln. Der enge Bezug zur Vergangenheit ist das Konzept, das auch auf die Liebesbeziehung übertragen wird. Das Liebespaar klammert sich an jedes Ding, das als Symbol ihrer Vergangenheit fungiert. In das Leben unmittelbar einzugreifen und die Vergangenheit durch eine solche Gestaltung mit konkreten Bildern immer wieder präsent machen zu können, ist ihr Lebenskonzept. Es erweist sich auch als ein selbstgenügsames und hermetisches Konzept, da das Liebespaar die Gegenwart als permanente Gestaltung der eigenen Vergangenheit wahrnimmt. Der Erzähler versucht, dieses Konzept auf ein Kind anzuwenden. Er ist bestrebt, das Gedächtnis des Kindes aktiv zu formen:

Ich redete auch viel mit dem Kind, fotografierte es jeden Tag und schaute dann, ob es sich schon verändert hätte. Außerdem glaubte ich, ich könnte mit dem Fotografieren bei dem Kind Bilder für die spätere Erinnerung hinterlassen, und stellte mir vor, auf diese Weise in den Erinnerungen des Kindes einmal vorzukommen. In der gleichen Absicht ging ich auch viel mit ihm herum, fuhr einmal mit ihm im Bus nach St. Louis hinein und stand lang am Mississippi-Ufer; der Geruch des Wassers würde dem Gedächtnis vielleicht nachhelfen. (Der kurze Brief 122, 123)

Durch die Erfahrung mit dem Kind kann der Erzähler Erinnerungen an die Kindheit wieder wachrufen. Außerdem kann er durch die Art, wie ein Kind in Amerika aufwächst, Parallelen zu seiner Entwicklung ziehen. Da er von negativen Bildern aus der Kindheit verfolgt wird, projiziert er oft die eigenen Zustände auf die Zustände des Kindes, so etwa das Klammern an alle Dinge, da das Persönliche noch nicht umgrenzt ist und ein Wechselbezug zur Umwelt noch nicht stattgefunden hat (vgl. Der kurze Brief 92). Auch die Erinnerung an den Zustand, durch ein nicht entwickeltes Bewusstsein allen Ängsten ausgeliefert zu sein, projiziert er auf das Kind (vgl. Der kurze Brief 109).

Andererseits kann der Erzähler durch die Erfahrung mit dem Kind lernen, indem er Vergleiche zieht. Die Zeichen, die er in seiner eigenen Kindheit wahrgenommen hat, hat er nie für selbstverständlich gehalten. Er hat sie hinterfragt und in seinen Vorstellungen nicht als endgültig akzeptieren wollen. $\mathrm{Er}$ hat schon immer nach dem 
Ursprünglichen gesucht und von anderen Zeichensystemen geträumt. Benedictine dagegen wächst in einem Zeichensystem ${ }^{3}$ auf, das eine Ersatzwelt für die Natur ist (vgl. Renner 1985: 81). Diese Zeichen und Bilder des modernen Lebens erlebt sie im Einklang mit der Realität (vgl. Brueggemann 1996: 156). Die Relation zwischen Zeichen und Objekt nimmt sie als naturgegeben wahr: „Daß das Kind hier sofort schon die Nachahmungen und Zeichen als etwas für sich anschaute, machte mich dann wieder fast eifersüchtig." (Der kurze Brief 125)

Die Kindheit des Erzählers war von naturnotwendigen Mechanismen gesteuert, so der kreisförmige Ablauf der Arbeiten in der Natur, um die Existenz zu erhalten. Benedictines Wahrnehmung ist dagegen von einer Fülle der Bilder des modernen Lebens überdeckt. Deswegen entwickelt Benedictine Sehnsüchte nach einem fixierten und vertrauten Raum, während der Erzähler, der in einem zu statischen Rahmen aufgewachsen ist, nach einem Ausbruch strebt. Die Dynamik des modernen Lebens überfordert die Wahrnehmung des Kindes (vgl. Der kurze Brief 93).

Durch die Begegnung mit Claire und ihrem Kind erweitert der Erzähler seinen Erfahrungshorizont. Ein anderes Zeichensystem, das in der amerikanischen Lebensform realisiert ist, ermöglicht ihm die Auseinandersetzung mit der eigenen Lebensform. Durch Claire kann er die Parallelen zu seiner Beziehung zu Judith ziehen. Das Kind wiederum ermöglicht die Vergleiche mit der Kindheit. Beides führt zu einer Bewusstseinsveränderung, die eine neue Positionierung des Ichs ermöglicht.

Die Bewusstseinsveränderung ist weiterhin von einer produktiven Deutung der Vergangenheit unmittelbar abhängig. Zu dieser Vergangenheit gehören auch die Figuren der Mutter und des Bruders. Der Erzähler telefoniert mit der Mutter und findet die Themen, die sie anspricht, lächerlich. Die Mutter spricht in allgemeinen Begriffen, die das Persönliche nicht berühren. Für den Erzähler gehört diese Lebensart zur Vergangenheit, und diese Einsicht ermöglicht die Befreiung aus dem aufgespielten Zeichensystem. Dem Bruder gegenüber verspürt er nicht einmal das Bedürfnis, ihn direkt

\footnotetext{
${ }^{3}$ Im engen Zusammenhang mit der Lebensform steht die Wahrnehmung der Zeichen im Umfeld, und die daraus resultierende Synthese dieser Zeichen in ein Zeichensystem, das umgekehrt die Existenz bestimmt. Für den Erzähler gelten die Zeichen der ursprünglichen Natur als wahre Wegweiser für eine den Menschen gerechte Existenz, die er hinter dem Schleier der Errungenschaften der Menschheitsgeschichte perzipiert und annimmt, während das Bewusstsein des Kindes durch die Wahrnehmung der sich unmittelbar aufdrängenden Zeichen der modernen amerikanischen Zivilisation bestimmt ist.
} 
anzusprechen. Er kommt zwar in die amerikanische Stadt Estacada, in der der Bruder lebt und als Holzarbeiter tätig ist. Nachdem er aber sein Zimmer besichtigt und das Verhalten des Bruders von Weitem beobachtet hat, ist inm klar geworden, dass der Bruder innerhalb eines des gleichen Zeichensystems seine banale Existenz nur fortschreibt, ohne jegliche Bewusstseinsentwicklung. So trennt sich der Erzähler auch von diesem Teil seiner Vergangenheit, zu dem weiter nichts zu sagen bleibt.

Der Raum ist wie die Figuren mit dem Thema der Bewusstseinsveränderung eng verknüpft. In Anlehnung an eine Aussage von Handke bespricht Manfred Mixner die Reise des Erzählers im Kontext einer Reise im Bewusstseins-Land (vgl. Mixner 145). Der Erzähler, ein österreichischer Schriftsteller, kommt nach Amerika und macht eine Reise von der Ost- zur Westküste. Allein dieses äußere Gerüst der Handlung deutet auf die Bedeutung der Bewegung hin. Der Raum Amerika, der im Bewusstsein der Amerikaner mit der Zeitgeschichte verschmolzen ist, ist eine neue Wirklichkeit, die dem Erzähler hilft, seine eigene Lebenslage zu hinterfragen. Indem er durch verschiedene Orte reist, nimmt er das Äußere zunächst als einen Anschauungsraum wahr:

Je mehr wir uns New York näherten, desto mehr wurden die Reklameschriften durch Bilder ersetzt: riesige überschäumende Bierkrüge, eine leuchtturmgroße Ketchupflasche, ein naturgroßes Bild von einem Düsenflugzeug über den Wolken [...]. (Der kurze Brief 31)

Dieser Anschauungsraum in Form einer zeichenhaften Landschaft bietet eine neue Perspektive und einen von der Vergangenheit abgetrennten Schutzraum. Der Erzähler fühlt sich in einem solchen Raum geborgen (vgl. Der kurze Brief 50, 51).

Die spontane Lebensart, die sich in einem solchen Raum reflektiert, schützt den Erzähler vor eingeübten Verhaltensnormen. Zugleich spiegelt sich dieser Raum in seinem Innern wider und bildet einen Kontrast zu beängstigenden Erlebnissen. Die ruckartigen Erlebnisse verschaffen immer wieder einen parallelen Raum, der als erinnerter Raum fungiert. Der erinnerte Raum erscheint in den plötzlichen Ausbrüchen der Angst und ist immer negativ konnotiert (vgl. Der kurze Brief 11, 12).

Durch Gegenüberstellung zweier Räume versucht der Erzähler den erinnerten Raum ins Bewusstsein zu rufen, ihn zu deuten, um einen Lösungsprozess in Gang zu setzen. Der Blick in die Ansätze einer Zukunft der Lösung wird im Hotelzimmer symbolisch angedeutet, ein geschlossener Raum, von dem aus der Erzähler durch das Fenster blickt: 
Vor dem Fenster, das in eine weite Parklandschaft mit kleineren Häusern hinausführte, standen hohe Birken. Die Blätter an den Bäumen waren noch klein, und die Sonne schien durch sie durch. Ich schob das Fenster hinauf, zog einen Lehnstuhl heran und setzte mich; die Füße legte ich auf die Zentralheizung, die vom Morgen noch ein bißchen warm war. (Der kurze Brief 14)

Die Sehnsucht, durch ein produktives Verhältnis zur Vergangenheit in eine traumhafte Zukunft hinauszublicken, wird hier symbolisch angedeutet. Ebenso eröffnet sich der gegenwärtige Raum als ein symbolischer im Erlebnis auf der Straße:

Ich ging die Vierundvierzigste Straße hinunter. 'Hinauf!' Ich kehrte um und ging in die andere Richtung. Ich mußte zum Broadway kommen, aber erst als ich die Avenue of the Americas und die Fifth Avenue schon überschritten hatte, merkte ich, daß ich in Wirklichkeit nicht umgekehrt war. Ich mußte es mir nur vorgestellt haben, umzukehren und in die andre Richtung zu gehen. Weil es mir aber vorkam, doch umgekehrt zu sein, blieb ich stehen und überlegte hin und her. Ich wurde schwindlig. (Der kurze Brief 36)

Der Drang, sich von den Leiden der Vergangenheit zu lösen und die Richtung der Bewusstseinsvorgänge zu ändern, wird an dieser Stelle räumlich veranschaulicht. Außerdem nimmt dieser Wunsch die Züge eines krampfhaften, ungeduldigen Verlangens an. Aus dem gegenwärtigen Raum, der die Geborgenheit reflektiert, wird der Erzähler immer wieder in den erinnerten Raum ruckartig zurückgeworfen. Dabei verharrt er darin in langen selbstanalytischen Phasen, die die Zeit ewig erscheinen lassen. Die inneren Wandlungen wiederum spiegeln sich im gegenwärtigen Raum, sodass dieser an manchen Stellen als gestimmter Raum zu erkennen ist. Er zeigt sich als eine Projektion der inneren Verfassung und verweist auf die Sorge und verschiedene Angstzustände:

Jetzt erst ging hier der Vollmond auf, und die weißen Bänke und Büsche standen ringsherum wie Erscheinungen. In einer Laterne war das Glas zerbrochen, eine Motte flatterte darin, bis sie verbrannte. Das Mondlicht war sehr hell, und doch nicht hell genug, so daß man zu platzen glaubte. Mein Herz schlug schmerzhaft, und ich seufzte oft, wenn ich Atem holte. (Der kurze Brief 101)

Die Bedrückung und der Schmerz, die das Innere beinahe zum Platzen bringen, projizieren die innere Spannung auf die Umgebung, die, dem Zustand entsprechend, von Hitze bedrückt erscheint. Solche Passagen erscheinen als Zustandsstationen, die mit der Fortbewegung des Erzählers in prozesshafte Umwandlungen im Bewusstsein überführt werden. So korrespondiert die Bewegung durch 
verschiedene amerikanische Orte mit der allmählichen Lösung von der Vergangenheit. Der Raum Amerika bietet durch das neue Zeichensystem, das Geborgenheit und Distanz symbolisiert, die Möglichkeit, die Verhaltensformen in dem erinnerten Raum erneut zu hinterfragen und zu ändern. Der Erzähler nutzt diese Chance:

[I]ch hob den Fuß, denn um die Schuhe hatte sich schon Wasser angesammelt, und tat einen großen Schritt zur Seite: das Bein versank sofort bis zum Knie in einem warmen Schlamm, [...] Ich fing an, das Bein herauszuziehen, und sprang schon, bevor ich es ganz aus der Erde hatte, zu den Bäumen zurück, so daß der Schuh im Schlamm unten steckenblieb [...] Nach einiger Zeit machte Claire die Augen auf, als ob sie sich nur schlafend gestellt hätte, und sah sofort, daß mir ein Schuh fehlte, und die Schlammkrusten an meiner Hose. Als ob sie einen Traum erzählte, sagte sie, was mir zugestoßen war, und ich bestätigte es. 'Hast du Angst gehabt?' - 'Es war eher eine Art Wut', antwortete ich. (Der kurze Brief 96, 97)

Durch solche Passagen verwandelt sich der gegenwärtige Raum in einen Aktionsraum, in dem der Erzähler in der Umgebung tätig wird. Das Verhaltensmuster eines Ausgeliefertseins in dem erinnerten Raum ändert sich dadurch, wenn auch die Teile der Vergangenheit weiterhin an der Seele haftenbleiben, mit Schlammkrusten symbolisch angedeutet. $\mathrm{Ob}$ man sich mit der Umgebung unmittelbar auseinandersetzt, in sie eingreift oder Distanz gewinnt ${ }^{4}$, deutet jede dieser Verhaltensformen auf eine produktive Umwandlung in Bezug auf die alten Verhaltensmuster hin. So ist der Raum nicht mehr von Passivität, Angst, Verboten und einer mechanischen Bewegung gekennzeichnet, sondern aufgrund der neuen Umgebung zu einem Hintergrund im Bewusstsein des Erzählers geworden, vor welchem ein neuer Entwurf des Raums gewonnen wird, der allmählich mythische Züge annimmt.

Der mythisch aufgeladene Raum entsteht aus dem Zusammenprall zwischen dem erinnerten und dem gegenwärtigen Raum. Der Aufbau der neuen Wirklichkeit lässt sich stufenweise verfolgen. Ihre Prophezeiung, wie der Name Providence signalisiert, lässt sich auch topografisch, da auf einer niederen Ebene sich spiegelnd, als eine Andeutung entziffern:

Im Hotel gab es im Keller neben dem Friseurladen eine Bar, wo ich dann im Dunkeln an einem Tisch Kartoffelchips aß; [...] Beim Würfeln passierte mir dann etwas Seltsames: ich brauchte gerade eine

\footnotetext{
${ }^{4} \mathrm{Vgl}$. die Erlebnisse in Oregon mit dem Mexikaner oder dem Betrunkenen. In: Der kurze Brief zum langen Abschied, S. 165, 186.
} 
bestimmte Zahl, und als ich den Becher hinkippte, blieben alle Würfel, bis auf einen, sofort liegen; während der eine aber noch zwischen den Gläsern durchrollte, sah ich an ihm die Zahl, die ich brauchte, kurz aufleuchten und dann verschwinden, bis der Würfel mit der falschen Zahl nach oben liegenblieb. Dieses kurze Aufleuchten der richtigen Zahl aber war so stark gewesen, daß ich es empfand, als ob die Zahl auch wirklich gekommen wäre, aber nicht jetzt, sondern zu EINER ANDEREN ZEIT. Diese andere Zeit bedeutete nicht etwa die Zukunft oder die Vergangenheit, sie war ihrem Wesen nach eine andere Zeit [...] Es war ein durchdringendes Gefühl von einer ANDEREN Zeit, in der es auch andere Orte geben mußte als irgendwo jetzt, in der alles eine andere Bedeutung haben mußte [...] (Der kurze Brief 27, 28)

Die nächste Stufe der Progression in Bezug auf den Aufbau des mythischen Raums findet sich in dem so oft zitierten Zypressenerlebnis (Der kurze Brief 100, 101). Das Zypressenerlebnis, in Anlehnung an die Symbolik von Trauer, Tod, aber auch Leben und Erkenntnis, verleiht dem Erzähler eine magische Erfahrung des Aufgehens im sanften Spiel der Natur und schenkt inm eine Wiedergeburt. Für einen kurzen Augenblick wird das Ich Teil der allumfassenden, wehmütigen Erkenntnis von ewigen, sanften Gesetzen des Aufnehmens und Scheidens, welche dem Persönlichen übergeordnet sind. Deswegen erlebt das Ich diese Erfahrung als eine Depersonalisation ${ }^{5}$. Als befreites Ich darf der Erzähler dieses Erlebnis nur kurz genießen, dafür aber eine neue Perspektive auf seine Selbstbezogenheit gewinnen, die ihm sowohl den Raum als auch die Zeit nicht als physische Größen, sondern als Ergebnis einer qualitativen Bewusstseinsveränderung anders erscheinen lässt. Die mythische Verschmelzung lässt sich weiterhin als eine sinnliche Erfahrung deuten, die sich allem Begrifflichen gegenüber, das für die alte Lebensform steht, als überlegen erweist. So wird die Wahrnehmung des Raums nicht von äußerlichen Größen, sondern von inneren Kräften abhängig. Der Erzähler sehnt sich nach einer Aufgabe seines alten Ichs, um die Umgebung mit einer anders gesteuerten Wahrnehmung genießen zu können. Die Loslösung von der Selbstfixierung und einem vorbestimmten Lebenskonzept wird somit zur Voraussetzung für eine sinnlich erfahrbare Existenz, die einen spontanen Blick auf das Andere und ein Zusammengefühl erlaubt. Die Erfüllung dieser Vorstellung ist im Garten von John Ford gegenwärtig,

\footnotetext{
${ }^{5}$ Der Terminus Depersonalisation ist der Analyse von Renner entnommen. Vgl. Rolf Günter Renner. Peter Handke. Stuttgart: Metzler, 1985.
} 
der in seinem paradiesisch gestalteten Raum ${ }^{6}$ eine mythische Erfüllung reflektiert und auch topografisch, auf einem Hügel erscheinend, die Vollendung eines stufenartigen Prozesses im Bewusstsein vermittelt. In diesem Garten kommt es zur endgültigen Fusion zwischen dem inneren und äußeren Raum. Der Regen, der Wind, die Tiere, das Gras und die Bäume erscheinen in einer friedlichen Übereinstimmung mit den Regungen in Menschen zu sein, von einem allgemeinen Lebensgefühl überströmt. Es wird dem Erzähler möglich, in der Umgebung geduldig und selbstverständlich zu existieren, sodass er in der Rolle eines entspannten Betrachters allein das Sehen als ein Erkennen ${ }^{7}$ erleben darf. Für die Authentizität der Fusion zwischen dem äußeren und inneren Raum bürgt die archetypische Figur eines Weisen.

\section{Zeit, Modus, Stimme}

Die Gestaltung der Zeit im Kurzen Brief zum langen Abschied macht deutlich, dass diese nicht durch eine bloße lineare Abfolge der Ereignisse gekennzeichnet ist. Sie wird durch häufige Analepsen unterbrochen, die einen bedeutenden Raum einnehmen und mit dem thematischen Schwerpunkt der Veränderung eng verbunden sind. Der Erzähler schwankt ständig zwischen der amerikanischen Gegenwart und seiner europäischen Vergangenheit. Die Reise in die Vergangenheit mischt sich immer wieder spontan und unabwendbar in die Gegenwart ein. Diese Anachronien sind subjektiv, denn sie ergeben sich aus den Gedanken des Erzählers. Dadurch wird der Eindruck erweckt, dass der Erzähler auch die vergangene Zeit nach Amerika mitgebracht hat, die seine Persönlichkeit zum großen Teil beansprucht, und dass er nun diese zu bewältigen versucht. Der Zusammenhang zwischen Ich und Zeit wird in einer Erinnerung an Judith, die dem Erzähler zufolge keinen Sinn für Zeit hatte, explizit thematisiert: „Dabei behindert andrerseits mich mein übertriebener Zeitsinn, dachte ich jetzt, und das heißt vielleicht: der übertriebene Sinn für mich selber, an der Gelöstheit und Aufmerksamkeit, die ich erreichen möchte." (Der kurze Brief 24)

Die Schlussfolgerung, die sich aus der Erinnerung an Judith ergibt, ist ein Beispiel dafür, wie die Analepsen in der Geschichte den Erzähler immer weiter zu neuen Einsichten führen. Ihm wird an dieser

\footnotetext{
${ }^{6}$ Zur Bedeutung von Landschaftsbildern, die auch in Handkes späteren Werken als Visionen der Erkenntnis von einem friedlichen Raum existieren vgl. die Analyse von Hans Höller. Eine ungewöhnliche Klassik nach 1945. Das Werk Peter Handkes. Frankfurt am Main: Suhrkamp, 2013, 125.

${ }^{7}$ Zu der Analyse der sinnlichen Erkenntnis vgl. Manfred Durzak. Peter Handke und die deutsche Gegenwartsliteratur. Stuttgart: Kohlhammer, 1982, 116.
} 
Stelle klar, dass die krampfhafte Bindung an die physische Zeit ihn von seinem Selbstsein nur entfernt hatte.

Der Erzähler kommt zunächst in ein Hotel in Providence. Der Zeitpunkt wird genau angegeben, es war Ende April, und er verrichtet dort die belanglosen Aktivitäten eines Hotelgastes. Dabei ist er in jedem Augenblick bedroht, von der Vergangenheit eingeholt zu werden. Er erinnert sich immer wieder an die alten Ängste, vor allem an Judith, seine Ehefrau, und an die Kindheit, die als bedrohlich und furchterregend in seinen Erinnerungen aufscheint. Die Ängste projiziert er nun auf die Gegenwart und verharrt dabei in langen Selbstanalysen. Diese sind zum einen durch den Bewusstseinsbericht wiedergegeben, der eine distanzierte Beobachtung seines Selbst zum Ausdruck bringt, und zum anderen werden die Selbstanalysen, durch Analepsen initiiert, mit inneren Monologen und Gedankenzitaten ausgefüllt. Sie werden somit auch durch den Modus als lange Sequenzen markiert. Die Selbstgespräche wiederum ermöglichen dem Erzähler, seine Selbstheit zu artikulieren und seelisch nicht auszugleiten, wie an dieser Stelle deutlich wird:

Der Boden der Badewanne war kreuz und quer mit breiten, hellen Streifen ausgelegt, die Heftpflastern ähnlich sahen und das Ausgleiten verhindern sollten. Zwischen dem Anblick dieser Heftpflaster und dem Gedanken an die Selbstgespräche ergab sich sofort eine Übereinstimmung, die so unverständlich war, daß ich zu kichern aufhörte und ins Zimmer zurückging. (Der kurze Brief 14)

Nach langen selbstanalytischen Phasen betrachtet der Erzähler allmählich die Zeit nicht als physische Größe, sondern er verinnerlicht sie qualitativ, was dazu führt, dass die Zeit im Werk nicht nur die Gestaltung des Diskurses bestimmt, sondern auch thematisch aufgegriffen wird. So hofft der Erzähler am Anfang, in seiner Einsamkeit verharrend, dass er sich schon verändert hat, und muss feststellen, dass die Veränderung durch eine bloße Abfolge der Zeit nicht möglich ist:

'Jetzt bin ich den zweiten Tag in Amerika', sagte ich und ging vom Gehsteig auf die Straße hinunter und auf den Gehsteig zurück: 'Ob ich mich schon verändert habe?' Ohne es zu wollen, blickte ich im Gehen über die Schulter und schaute dann geradezu ungeduldig auf die Armbanduhr. (Der kurze Brief 20)

Ebenso heißt es an einer anderen Stelle:

Dieses Entsetzen und das Bedürfnis, möglichst schnell anders zu werden und es endlich los zu sein, machten mich ungeduldig. Die Zeit verging mir so langsam, daß ich schon wieder auf die Armbanduhr schaute. Der wohlbekannte hysterische Zeitsinn stellte sich ein. Vor 
Jahren hatte ich einmal eine dicke Frau im Meer baden sehen und alle zehn Minuten zu ihr hingeschaut, weil ich allen Ernstes glaubte, sie müßte doch inzwischen schlanker geworden sein. Und jetzt in der Snackbar blickte ich immer wieder zu einem Mann mit einer verkrusteten Wunde an der Stirn, weil ich wissen wollte, ob die Wunde nun endlich verheilt war. (Der kurze Brief 22, 23)

Die Einsicht, dass die wahren Veränderungen durch eine Verinnerlichung der Zeit und deren Umsetzung in die qualitative Größe geschehen, entwickelt sich im Laufe der Geschichte zu einer engen Verknüpfung zwischen dem Drang nach Veränderung des Ichs und der Auffassung der Zeit.

So wird dem Erzähler die Zeit in den Hotelzimmern zu lang, denn er kann aus seiner alten Selbstheit nicht spontan ausbrechen, was inn verzweifeln lässt. Diese psychische Lage wird zusätzlich durch die Erzähldauer veranschaulicht, denn im ersten Teil der Geschichte ist eine Dehnung der Erzählzeit sehr offensichtlich. Jeder einzelne Tag bis zum Treffen mit Claire wird detailliert geschildert, die Zeitangaben zu den einzelnen Tagen werden angegeben, und nur der erste Tag in Amerika umfasst beispielsweise neun Seiten. Diese Dehnung bekommt im ersten Teil der Geschichte beinahe das Ausmaß eines Zeitstillstands. Die Gestaltung der Zeit ändert sich durch die Begegnung mit Claire und ihrem Kind. Erst von dieser Stelle an ist eine Raffung der Erzählzeit zu beobachten (vgl. Krajenbrink 113). In dieser Zeitphase, in der er einen intensiven Kontakt mit anderen Menschen hat, erlebt der Erzähler die Gegenwart gelassen und ist währenddessen von dem "hysterischen Zeitsinn“ (Der kurze Brief 22) befreit. Die Selbstgespräche, die zu Anfang der Geschichte dominieren, gehen nun in Dialoge mit Claire über, die auch zur Analyse seines Selbst dienen und die in Bezug auf die Dauer als direkte Figurenrede eine Deckung zwischen der erzählten Zeit und der Erzählzeit aufweisen. Die Deckung gibt den Anschein von Unmittelbarkeit wieder, und die komplexen Selbstbeobachtungen aus dem ersten Teil werden dadurch deutlich aufgelockert. Der Wechsel des Ortes ermöglicht dem Erzähler eine neue Lebensweise und damit einen neuen Zeitsinn zu entdecken. Er sagt an einer Stelle: „'Ich bemerke, wie sich bei mir in Amerika jetzt die Kindererlebnisse wiederholen', sagte ich. 'Alle Ängste, Sehnsüchte stellen sich wieder ein, die ich schon längst hinter mir glaubte'“ (Der kurze Brief 101, 102).

Erst eine andere Gegenwart ruft wieder die Vergangenheit hervor, die durch Distanz und Vergleiche produktiv aufgearbeitet werden kann. Das war auch das Ziel der Reise, was noch einmal Claire unterstreicht: „Du bist hierhergekommen wie mit einer Zeitmaschine, 
nicht um den Ort zu wechseln, sondern um in die Zukunft zu fahren" (Der kurze Brief 85).

Der Erzähler genießt die Zeit mit Claire auch bei dem Liebespaar, das eine amerikanische Lebensweise verkörpert. In diesem Abschnitt ist eine Raffung der Zeit zu beobachten, und die genauen Zeitangaben werden ausgelassen. Dadurch erscheint diese Passage als ein utopischer Chronotopos ${ }^{8}$, dessen Länge, im Unterschied zu den ersten drei Tagen in Amerika, nicht zu rekonstruieren ist. Nach dem Abschied von Claire verharrt der Erzähler in der Einsamkeit, und die einzelnen Zeitangaben werden wieder markiert: „An diesem ersten Tag in Tucson verließ ich nicht mehr das Hotel. [...] In der Nacht träumte ich viel. [...] Zu Mittag fuhr ich mit dem Taxi zum Flugplatz [...]" (Der kurze Brief 166,168).

Der verlangsamte Erzählrhythmus zeigt an, dass der Erzähler nach der Trennung von Claire der Zeit wieder ausgeliefert ist und damit seinem Selbst, das er weiterhin bewältigen möchte:

'Die Zeit schleifte so hin.' Dieser Satz aus einer Geschichte von Adalbert Stifter fiel mir ein. Ich setzte mich auf und nieste. Plötzlich kam es mir vor, als hätte ich dabei ein ganzes Stück Zeit übersprungen. Ich wünschte mir nun, daß mir so bald wie möglich etwas zustoßen würde. (Der kurze Brief 167, 168)

Der Erzähler bewegt sich also ständig zwischen Gegenwart und Vergangenheit, zieht dabei Vergleiche, registriert einzelne Veränderungen an sich und nutzt somit den Wechsel des Ortes bzw. die Bewegung zu einer qualitativen Veränderung seines Ichs. Ein solcher Zeitsinn unterscheidet sich von einem unproduktiven Zeitsinn, wie inn seine Mutter vermittelt: „Sie gehe jetzt viel herum und vergesse dabei ganz auf die Zeit" (Der kurze Brief 178).

Während die Mutter die Bewegung nutzt, um eine Ablenkung von sich selbst zu erreichen, geht der Erzähler anders damit um. Seine Distanz zu ihrer Lebensweise wird an dieser Stelle zusätzlich durch transponierte Rede, hier indirekte Rede, unterstrichen. Auch der Bruder ist nicht imstande, einen anderen Zeitsinn zu entwickeln, sondern verharrt in der Vergangenheit, die er ohne jegliche Reflexion als selbstverständlich erlebt.

Die Analyse der Zeitebene zeigt, dass sie von häufigen Analepsen gekennzeichnet ist, die eine chronologische Ordnung unterbrechen. Sie haben die Funktion, einen Vergleich zwischen

\footnotetext{
${ }^{8}$ Der Chronotopos als Utopie ist der Analyse von Aminia M. Brüggemann entnommen. In: Chronotopos Amerika bei Max Frisch, Peter Handke, Günter Kunert und Martin Walser. New York: Peter Lang, 1996.
} 
Gegenwart und Vergangenheit zu ziehen, um zu einem neuen Ich zu gelangen. Dadurch wird die Zeit auch thematisch behandelt, da sie mit Ich und Sinn gleichgesetzt werden kann, wie es aus den erwähnten Beispielen zu erschließen ist. Die qualitative Auffassung der Zeit kommt auch in plötzlichen Erleuchtungen zum Ausdruck. Sie legen zusätzlich eine dritte Zeitebene fest, die als Produkt des Umtausches zwischen Gegenwart und Vergangenheit betrachtet werden kann. Sie vollzieht sich in Augenblickserfahrungen. Die Augenblickserfahrungen kann man als qualitative Zeitpausen bezeichnen. Durch diese epiphanischen Augenblicke, die zu einem Zeitstillstand führen, zeigen sich eigentlich die qualitativen Veränderungen im Innern des Erzählers als plötzliche Erleuchtungen. Ein Beispiel dafür wäre das Zypressenerlebnis, das auf eine Befreiung von Selbstbezogenheit hindeutet. Die qualitativen Zeitphasen, die zu einem anderen Umgang mit sich selbst und der Umwelt führen, ereignen sich momenthaft. Ein weiteres Beispiel dafür ist die Erfahrung mit Claire:

Jene ANDERE ZEIT, die ich in Providence bei dem kurzen Aufblitzen des Würfels erfahren hatte, erstreckte sich nun vor mir als eine andere Welt, die ich nur zu betreten brauchte, um meine angstanfällige Natur und ihre Beschränktheiten endlich loszusein. (Der kurze Brief 106, 107)

Die Augenblickserfahrung verweist auf die Einsicht, dass jene andere Zeit, die für eine andere Wahrnehmungsweise steht, eine Frage der inneren Umstellung ist und somit vom Erzähler selbst abhängt. Diese innere Umstellung wird an einer weiteren Stelle deutlich:

Schließlich war es Claire, die in einer Geschichte von einem Schwein und einer Kutsche, die ich gerade mit klopfendem Herzen erzählte, auf einmal so ernst wurde, daß ihr Gesicht sich sofort bis zur Unkenntlichkeit veränderte. Früher wäre mir das vielleicht als Ausbruch eines Wahnsinns vorgekommen, an diesem Abend aber erlebte ich es mit einer fast vergessenen Lust an früherer Feierlichkeit als den Augenblick einer Wahrheit, die meinen Wahnsinn, der darin bestand, daß ich fürchtete, ein andrer mir gegenüber könnte plötzlich wahnsinnig werden, für immer lächerlich machte. (Der kurze Brief 109)

Wegen der qualitativen Zeitphasen, die sich aufdrängen, findet der Erzähler die Lösung nicht in einer Entpersönlichung, sondern in der Aufgabe der ultimativen Selbstbezogenheit, die ermöglicht, ein erleuchtetes Bewusstsein von sich selbst zu gewinnen und dadurch einen offenen Blick für die Umwelt zu entwickeln. Diese Einsicht ist auch der Grund dafür, dass der lange Bericht des Erzählers im Garten des Filmregisseurs John Ford, durch direkte Figurenrede ergänzt, sich nicht mehr auf die Selbstanalysen bezieht, sondern, da der Erzähler im Einklang mit Ich und Welt ist, ein gelassenes Bild der Umwelt entwirft. 
Der Erzähler spielt eine zweifach bedeutende Rolle, da er auf der Ebene des Diskurses als Stimme fungiert, die Erzählinstanz, die die Geschichte vermittelt, und auf der Ebene der Geschichte als Hauptfigur. Der autodiegetische Erzähler bekommt somit eine komplexe Rolle in der Erzählung. Die Geschichte wird von ihm vermittelt, und die interne Fokalisierung wird durch die Geschichte beibehalten. Die einzigen Figuren, die im Hinblick auf die Fokalisierung etwas Raum bekommen, sind die Figur Claire, durch deren Sicht erreicht wird, dass die Veränderungen am Erzähler auch von außen artikuliert werden, und die Figur John Ford, dessen Rede zur Bestätigung von Einsichten des Erzählers dient. Somit weicht diese Übergabe der Fokalisierung von der anfänglichen Absicht, die ganze Geschichte durch eine interne Sicht in ihrer Totalität zu erfassen, nicht ab. Der Erzähler bedient sich zu einem großen Teil der Geschichte des Berichts. Die vorherrschende Wahl dieses Modus zeigt, dass der Erzähler, obwohl selbst ein Teil der diegetischen Welt, auf eine Objektivität hinauszielt. Auch der Umstand, dass er als ein erinnertes Ich auftritt, spricht dafür. Da er sich zwischen Gegenwart und Vergangenheit bewegt, kommt in den langen Erinnerungspassagen ein erinnertes Ich zu Wort. Dadurch wird eine Distanz zu den Geschehnissen erreicht, aber auch der Eindruck der objektiven Vermittlung erweckt. Der Erzähler bekommt somit paradoxerweise die Merkmale einer auktorialen Sicht, obwohl sich diese Absicht in Bezug auf die ontologische Stellung eines autodiegetischen Erzählers nicht bewähren kann. Der dramatische Modus, der stellenweise in die Erzählung eingebettet ist, so das Gedankenzitat und der innere Monolog, lässt die distanzierte Haltung, durch den Modus des Berichtes erreicht, glaubwürdig erscheinen. Durch den Einbezug des dramatischen Modus wird außer der Glaubwürdigkeit der Vermittlung der Anschein von Unmittelbarkeit erreicht, was die Geschichte intensiviert und vertrauter macht. Der innere Monolog ist beispielsweise an der folgenden Stelle sichtbar: „Wo aber war die Umgebung, in der ich endlich zeigen würde, daß ich anders sein konnte?" (Der kurze Brief 20) So ist es auch beim Erlebnis des Würfelspiels: „Mein Leben bis jetzt, das durfte noch nicht alles sein!“ (Der kurze Brief 28) Die unmittelbare Bewusstseinsvermittlung wird auch durch Zitate intensiviert. So grübelt der Erzähler im Hotelzimmer: „'Woher hat sie das Geld für die Reise?' fragte ich. [...] 'Der Brief war sehr lang unterwegs', sagte ich. 'Ob sie inzwischen tot ist?'“ (Der kurze Brief 15$)^{9}$

\footnotetext{
${ }^{9}$ Die Hervorhebungen des Autors sind im Kursiv angeführt. Auch die Hervorhebungen
} 
Oder an einer weiteren Stelle: „'Jetzt bin ich den zweiten Tag in Amerika', sagte ich und ging vom Gehsteig auf die Straße hinunter und auf den Gehsteig zurück: 'Ob ich mich schon verändert habe?'“ (Der kurze Brief 20)

Die zitierten Stellen sind nicht als Gedankenzitate wiedergegeben, da innen kein verbum credendi folgt, sondern als Selbstgespräche bzw. Monologe, da sie konsequent durch ein verbum dicendi ergänzt werden (in den angeführten Beispielen in Fettdruck angegeben). Dieser Umstand lässt sich durch die kommunikative Ebene erklären, da, obwohl es sich um den Bewusstseinsinhalt handelt, der Absender und zugleich der Adressat der Erzähler ist. Aus diesem Grund erscheinen sie als Monologe, im Sinne des dramatischen Modus der ausgesprochenen Gedanken, werden aber merkwürdigerweise von der Figur vermittelt, die sie ausspricht, was wiederum auf den Erzähler als erinnertes Ich zurückzuführen ist. Die Selbstgesprächszitate als artikulierte Gedanken einer Figur, die mit dem Adressaten zusammenfällt, sind als Monologe eher für eine dramatische Struktur üblich. In einem Erzählwerk, bei dem die narrativen Techniken ermöglichen, in das Innere der Figur durch den inneren Monolog und die Gedankenzitate zu gelangen, sind die dramatischen Monologe im traditionellen Gebrauch nicht üblich. In dieser Erzählung sind sie aber häufig vorzufinden.

Der dramatische Modus tritt auch nach der Begegnung mit Claire auf, obwohl auch dieser Teil der Geschichte im Bericht vorherrschend vermittelt wird. Er zeigt sich in Gesprächen mit Claire, die in der direkten Rede wiedergegeben werden, was als Ersatz für die unmittelbare Bewusstseinsvermittlung aus dem ersten Teil fungiert. Auch in diesem Teil der Geschichte wird eine Unmittelbarkeit, Vertrautheit und Glaubwürdigkeit der Geschichte erzielt, was mittels der Einschübe des dramatischen Modus auch gelingt.

Der Erzähler ist, wie schon erwähnt, nicht nur die Erzählinstanz, sondern auch die Hauptfigur. Als Träger der Handlung hat er sich auf der Reise durch Amerika eine klare Aufgabe gestellt - er strebt nach einer Bewusstseinsveränderung. Diese Veränderung, die zunächst eine Loslösung bedeutet, bezieht sich auf die Angstzustände, denen er vor allem in der Kindheit ausgeliefert war, ferner auf eine begriffliche Welt, in der alles sprachlich im Voraus gedeutet ist, und schließlich auf ein Rollenleben, so wie es sich in der Ehe mit Judith vollzogen hat. Alle diese bewussten und unbewussten Leiden schlugen beim Erzähler in eine paranoide Selbst-Fixiertheit um. Die physische 
Trennung von den alten Lebensformen ermöglicht ihm, Distanz zu gewinnen und durch eine neue Lebensform seine Zustände reflexiv aufzuarbeiten. Die Veränderungen in seinem Bewusstsein verlaufen in verschiedenen Formen und Stadien. Manfred Mixner zählte in seiner Untersuchung die Reaktionen zu den Formen der Bewusstseinsveränderung auf, die nun im Gegensatz zu Bloch dem sozialen Umfeld angepasst werden (vgl. Mixner 147): die Verhaltenssteuerung (vgl. ebenda: 148), die bewusste Beobachtung des eigenen Reagierens auf die Umwelt (ebenda) und die Identifikationspunkte, die er beim Lesen findet (ebenda 150). Der Erzähler versucht in seinen langen Reflexionen die alten Angstzustände, die sich in der leidenden Erinnerung immer wiederholen, in eine tätige Erinnerung umzuwandeln. Er benennt sie, versteht sie und ändert durch neue Erfahrungen den Umgang mit ihnen. Dabei helfen ihm Kontakte mit neuen Menschen, vor allem mit Claire und mit dem Liebespaar. Die Einstellung zur Vergangenheit ändert sich dadurch wie das Verhalten in der Gegenwart. Ein Beispiel wäre sein Verhältnis zur Natur. Er fühlt sich schon von Kindheit an von ihr bedroht, ändert aber bei dem Schlammerlebnis seinen Umgang mit der Natur, indem er aktiv eingreift. Er rettet sich aus dem Schlamm, und der Mann, der sie als Landesbesitzer verfolgt, eine Verkörperung der alten Ängste vor den Fremdbesitzern, schrumpft vor seinen Augen als ein archetypischer Vertreter des Ungeheuers in einem grotesken Bild zusammen. Durch den neuen Umgang mit der Umwelt wird der Erzähler immer selbstbewusster. Die Konsequenz dessen ist, dass die alte Lebensform von allein auseinanderfällt. Das zeigt sich in der Szene mit Judith, die als Verkörperung aller Verfolgungen bei dem Versuch, den Erzähler umzubringen, von selbst zusammenbricht. Der Erzähler erlebt seine alte Lebensform als Gefangenschaft in einer begrifflichen Welt, in der jede Art einer individuellen Existenz durch schematische Deutungen typisiert wird. Er sagt an einer Stelle:

Ein heftiger, dann lustiger Ekel vor allen Begriffen, Definitionen und Abstraktionen, in denen ich gerade gedacht hatte, ließ mich beim Hinausgehen kurz stehen bleiben. [...] Ein Student mit kurzen Haaren, pausbäckig, in Bermudahosen, mit dicken Schenkeln, in Turnschuhen, kam mir draußen entgegen, und ich blickte ihn entsetzt an, fassungslos bei dem Gedanken, daß jemals jemand es trotzdem wagen könnte, etwas Allgemeines über diese einzelne Figur zu sagen, daß jemand ihn typisieren und zu einem Vertreter von etwas anderem machen würde. (Der kurze Brief 24)

Sein ultimativer Anspruch auf eine persönliche Entwicklung war eine lange Zeit eben durch diese Schematisierungen verhindert. In 
einer Gesellschaft, in der jede Erscheinung sprachlich auf einen vorgedeuteten Begriff gebracht wurde, war auch jede zwischenmenschliche Beziehung dazu verurteilt, ein Rollenverhalten zu sein. In der Schilderung der Beziehung mit Judith, in der jeder authentischer Austausch unmöglich geworden war, spricht der Erzähler ein vorgespieltes Rollenleben noch einmal an:

Wenn wir nicht mit uns selber allein waren, sondern Rollen spielten, die von Gastgebern im Restaurant, Reisende am Flughafen, Kinobesucher, Gäste, und auch von den anderen als Verkörperungen von Rollen behandelt wurden, vertrugen wir uns wieder, weil wir uns ganz als Rollenträger erlebten, und waren fast stolz, wie selbstverständlich wir diese Rollen schon spielten. (Der kurze Brief 136, 137)

Der Erzähler will sich vom Rollenverhalten unbedingt trennen und zieht den authentischen Wechselbezug diesem vor. Durch die authentischen Erlebnisse kommt er auch zu einer neuen Form der Erkenntnis, die das Produkt eines offenen Kontakts mit der Umwelt wird und sich in einer sinnlichen Erfahrung offenbart, weit von den rationalen Begriffen und Definitionen entfernt. Die neue Art der Erkenntnis zeigt sich sowohl im Zypressenerlebnis als auch auf dem Dampfschiff, auf dem das Geräusch ein Einverständnis zwischen Ich und Welt hervorruft. ${ }^{10}$

Dem Erzähler gelingt es, in der neuen Umgebung spontaner zu werden, so kann er Claire ganz selbstverständlich bei dem Kind helfen. Ein freier, unmittelbarer Umgang mit der Umwelt wird immer deutlicher. Auch die Loslösung von einer extremen Selbstbezogenheit gelingt dem Erzähler. Diese Veränderung wird schon im Gespräch mit Claire angedeutet. Er unterbricht immer wieder seine Rede, sobald er bemerkt, dass er wieder von sich selbst spricht (vgl. Der kurze Brief 74). Die Selbstbezogenheit geht allmählich in einen flexiblen Umgang mit der Umwelt über. So wird der Erzähler am Ende der Geschichte zu einem veränderten Ich.

Die persönliche Entwicklung des Erzählers bleibt aber nicht Selbstzweck. Eine tiefere Problematik, die unter der persönlichen Entwicklung weitergesponnen wird, offenbart sich in seiner schriftstellerischen Existenz. Der Erzähler lernt aus der neuen Lebensform, die Amerika heißt, und setzt sich zugleich intensiv sowohl mit der klassischen Lektüre als auch mit der gegenwärtigen Popkultur

\footnotetext{
${ }^{10}$ Die sinnliche Erkenntnis im Kurzen Brief zum langen Abschied behandelt ausführlich Manfred Durzak in seiner Untersuchung. Durzak deutet die sinnliche Erkenntnis, die man einer begrifflichen Orientierung vorzieht, als ein Zeichen der modernen Literatur. In: Manfred Durzak: Peter Handke, S. 115.
} 
auseinander. Beide Sphären tragen zu seiner Veränderung bei, mit dem Ziel, die persönliche Erfahrung in poetologische Modelle umzusetzen. Alle Veränderungen sollten zu neuen Schreibmodellen führen, welche das Authentische in die Fiktionalität umsetzbar machen würden. Demzufolge verzichtet der Erzähler zum Beispiel darauf, Rollen zu schreiben:

'Es fällt mir schwer, Rollen zu schreiben', antwortete ich. 'Wenn ich jemanden charakterisiere, kommt es mir vor, als ob ich ihn damit entwürdige. Aus allem Besonderen an einer Figur wird dann ein Tick. Ich spüre, daß ich anderen nicht so gerecht werden kann wie mir selber. Wenn ich Leute auf der Bühne reden lasse, schnappen sie mir schon nach den ersten Sätzen zu und sind für immer auf einen Begriff gebracht. Deswegen werde ich vielleicht lieber Geschichten schreiben'. (Der kurze Brief 158)

Eine weitere poetologische Konsequenz seiner persönlichen Veränderung ist, dass er durch den flexiblen Austausch zwischen Ich und Welt einen offenen Blick für die Umwelt gewinnen kann. Der Erzähler nimmt die Außenwelt nicht mehr selbstbezogen wahr und wird immer mehr imstande, die Umwelt, so wie sie sich präsentiert, zu beschreiben. Diese Veränderung, die unmittelbar einen Eingang in das Schreiben findet, zeigt sich in dem Wechsel der Stimme. Statt einer IchStimme meldet sich eine unpersönliche „man“-Stimme, welche die Umwelt nur registriert: „Ich bemerkte, daß ich zum ersten Mal seit langem wieder jemanden ohne Anstrengung aus der Nähe betrachten konnte. Man ${ }^{11}$ sah den Soldaten an" (Der kurze Brief 59).

Schließlich kann der Erzähler, da er einen harmonischen Zustand zwischen Ich und Umwelt erreicht hat, von den Geschehnissen mit John Ford als ein Betrachter berichten und endlich selbst in die Fiktion eingehen. Die Geschichte einer persönlichen Entwicklung ist für eine fiktionale Bearbeitung tauglich. Auf Handkes poetologische Projektion, jede Lebenserfahrung als Innenwelt des Schriftstellers in ein Schreibverfahren als Außenwelt umzusetzen, verweist Kurt Batt in seinem Beitrag zu Handkes Erzählung $(617,618)$.

\section{Schlussfolgerung}

Der kurze Brief zum langen Abschied unterscheidet sich von Handkes vorigen Werken vor allem darin, dass der Fokus nicht mehr auf der Auflösung der traditionellen narrativen Strukturen liegt, und dass ein Lösen von dem Einfluss des Nouveau Roman zu beobachten ist. Kennzeichnend für den Kurzen Brief ist ferner der Umstand, dass

${ }^{11}$ Die angeführte Hervorhebung im Kursiv ist dem Original entnommen. 
einer privaten Geschichte der Eingang in die Literatur ermöglicht wird. Daher ist das Werk entscheidend biografisch gefärbt. Was in den 1960er-Jahren als Kritik der traditionellen Erzählweise zu erkennen war, ist in dieser Erzählung durch die Umsetzung des Privaten und Authentischen in ein fiktionales Werk zu einer neuen Schreibmethode geworden, die schließlich einen neuen narrativen Umgang mit der Geschichte mit sich gebracht hat.

Das vorherrschende Thema, das Handke in den Werken der 1970er-Jahre aufarbeitete - die Trennung vom Lebenspartner - setzt in dieser Erzählung ein. Im Kurzen Brief zum langen Abschied ist es die Trennung von Judith, in Die Stunde der wahren Empfindung die Trennung von Stefanie, in Die linkshändige Frau die Trennung von Bruno. Die Auflösung der Ehe erweist sich auf der Ebene der Tiefenstruktur als initiales Moment für eine umfassendere Trennung von den auferlegten sozialen Rollen, welche die Entwicklung des Individuums von Kindheit an prägen. Aus diesem Grund wird die Trennung vom Lebenspartner zugleich als Trennung von der Kindheit problematisiert, was in Der kurze Brief zum langen Abschied, aber auch in Die Stunde der wahren Empfindung zum Ausdruck kommt. Die Trennung wird ferner mit der Problematik der Suche nach der wahren Identität eng verknüpft. Diese Problematik wird in den 1970er-Jahren mit der Erzählung Die linkshändige Frau abschließend apostrophiert.

Da die Trennung als thematischer Schwerpunkt erscheint, ist für die Erzählung Der kurze Brief zum langen Abschied, aber auch die Erzählungen Die Stunde der wahren Empfindung und Die linkshändige Frau kennzeichnend, dass die Geschichte in dem Moment beginnt, in dem die Trennung vom Lebenspartner in Gang gesetzt, und somit in medias res eröffnet wird. Danach wird eine Reihe von existenziellen Motivketten entfaltet, so die Einsamkeit, die Angst, der Traum, der Wunsch, die Desorientierung, die Wahrnehmung und die Auseinandersetzung mit dem eigenen Bewusstsein. Im Kurzen Brief und den zwei weiter oben erwähnten Erzählungen aus den 1970erJahren, welche die Trennung als Einschnitt im Leben der Figuren vermitteln, wird die Handlung als eine Bewusstseinsreise präsentiert, die von der Hauptfigur initiiert wird. Somit erscheinen die Protagonisten in den erwähnten Werken als dynamische Figuren, die entwicklungsfähig sind. Die Handlung wird auf die inneren Wandlungen der Hauptfigur versetzt - im Kurzen Brief ist das der IchErzähler - sodass die Zustände, bzw. Bewusstseinsvorgänge verfolgt werden, was den Geschichten eine statische Struktur verleiht.

In den erwähnten Erzählungen wird dieses Merkmal umso mehr zugespitzt, als die biografischen authentischen Erfahrungen des 
Autors von den subjektiven Vorgängen im Innern der Figuren schwerlich noch zu trennen sind. Durch die Aufarbeitung der Trennung streben die Protagonisten nach einem authentischen Wechselbezug zwischen Ich und sozialem Umfeld, der nicht mehr von auferlegten gesellschaftlichen Mechanismen determiniert ist. Sie versuchen, eine neue Lebensform zu gewinnen, die die Individualität bewahrt und gleichzeitig eine Balance zwischen Ich und Welt ermöglicht. Diesen programmatischen Vorsatz behält Handke auch in späteren Werken bei, so in der Tetralogie Langsame Heimkehr, in Der Chinese des Schmerzes, Mein Jahr in der Niemandsbucht, usf. $^{12}$

Bei der Trennung vom Leben als einer von der Gesellschaft auferlegten Rolle ist weiterhin die Sprache ein wichtiger Bestandteil von Geschichten, die im Unterschied zu den 1960er-Jahren nicht mehr als auferlegter Mechanismus in der Erzählpraxis bloßgestellt, sondern als gesellschaftlicher Mechanismus entlarvt wird, der jeden Lebensschritt eines Einzelnen vorbestimmt.

Der Zweifel an der vorgegebenen Sprache und ein aufmerksamer Umgang mit ihr wird zur Voraussetzung für die Befreiung von der auferlegten Identität und die Artikulierung einer authentischen Existenz. Die umrisshafte Artikulierung der neugewonnenen Persönlichkeit deutet ferner auf die Einstellung hin, man könne sich dem Authentischen eines Individuums durch die Sprache nur annähern. ${ }^{13}$

Die Aura des Geheimnisvollen um die Identität wird in Handkes Werken stets beibehalten. Die Sprache gelangt nur teilweise in das Innere. Dem Zweifel an der sprachlichen Definierbarkeit der Persönlichkeit wird zudem die sinnliche Erfahrung gegenübergestellt. Der kurze Brief ist auch in diesem Sinne die Vorwegnahme einer sinnlich erfahrbaren Welt. Die sinnlichen Erlebnisse, die sich in augenblickhaften Erleuchtungen vollziehen, sind ein unumgänglicher Bestandteil von Handkes weiteren Werken, so in der Erzählung Die Stunde der wahren Empfindung, die diese Einstellung auch im Titel apostrophiert, in Die linkshändige Frau, in Die Lehre der Sainte-Victoire, Dem Bildverlust, usf. Da sie charakteristisch für Handkes Geschichten

\footnotetext{
${ }^{12}$ Zum thematischen Aspekt der Entwicklung und Bewahrung der Individualität in der dargegebenen Welt vgl. den Beitrag von Andjelka Krstanović „Die Erzählverfahren in Die linkshändige Frau“ (in Druck) In: Facta Universitatis, Series: Linguistic and Literatur, Vol. 18, No $1,2020$.

${ }^{13}$ Zu der Bedeutung der Sprache bei der Entwicklung der Individualität vgl. den Beitrag von Andjelka Krstanović „Authentizität und Fiktionalisierung. Narrative Überlegungen zu Peter Handkes Erzählung 'Wunschloses Unglück' "In: Sprachkunst. Beiträge zur Literaturwissenschaft, 2019/2, 2020, S. 231-254.
} 
sind, werden sie als epiphanische Augenblicke in einzelnen Untersuchungen näher analysiert (vgl. Durzak, 1982; Schmidt, 2008).

In der Welt der Figuren liegt der Schwerpunkt auf der Bewusstseinsentwicklung der Hauptfigur. Sie durchgeht einen mühevollen Prozess der Selbsterkenntnis, zu deren Voraussetzung die Befreiung von der auferlegten Rollenexistenz gehört. Die Selbsterkenntnis wird als erreichtes Ziel am Ende der Geschichte dargestellt, begrifflich aber nicht näher definiert. Sowohl der Kurze Brief als auch die weiteren Erzählungen aus den 1970-er-Jahren, so Die Stunde der wahren Empfindung und Die linkshändige Frau, enden in einer entspannten Atmosphäre, die durch die Verlegung der Handlung auf das äußere Geschehen erreicht wird. So genießt der Ich-Erzähler in Der kurze Brief zum langen Abschied das Gespräch zwischen Judith und John Ford, und rückt somit nach einer leidensvollen Bewusstseinsreise in die entspannte Rolle eines Betrachters.

Am Ende zeichnet sich also die Entspannung ab, die nicht durch die Zergliederung des Inneren, sondern durch die Verlegung der Darstellung auf das Äußere veranschaulicht wird, was die Auseinandersetzung mit der Selbstentwicklung als abgeschlossene Phase markiert. Sie fungiert als Zwischenphase und Andeutung eines neuen Anfangs und verleiht der Geschichte die Form eines offenen Endes. So wird ein weiterer programmatischer Vorsatz narrativ zum Ausdruck gebracht - die Selbstentwicklung wird als Prozess der steten Aneinanderreihung der fragmentarischen Erkenntnisphasen begriffen.

Die Welt der Figuren in Handkes Werken ist durch eine spezifische Einstellung zu Raum und Zeit wesentlich bestimmt. Dem Ich-Erzähler im Kurzen Brief dünkt, sich auf der Straße umgedreht zu haben, was mit der Veränderung der Gedankenrichtung assoziiert wird.

Der kurze Brief eröffnet in diesem Kontext die Entwicklung eines zielgerichteten Verhältnisses zu den Größen Zeit und Raum. Die Hauptfigur zeigt die Sehnsucht nach einem anderen Raum. Dabei ist kennzeichnend, dass sie auch ihre Bewusstseinsveränderung räumlich erlebt.

Beim Ich-Erzähler in Der kurze Brief, ferner bei Gregor Keuschnig in Die Stunde der wahren Empfindung und Marianne in Die linkshändige Frau erweist sich der andere Raum als ein verinnerlichter, der von Innen heraus durch eine neue Weltsicht den äußeren Raum qualitativ bestimmt. In der späteren Erzählung Langsame Heimkehr erkundet die Hauptfigur Sorger die Landschaftsformen, die er in einem simultanen Bild verinnerlicht, das zur existenziellen Erkenntnis führt. Auch in weiteren Werken wird die qualitative Auffassung des Raumes 
eine Konstante bleiben. Die Frage des Raumes wird stets zur Frage der neuen Wahrnehmung, die zur neuen Lebensform führt.

Ähnlich verhalten sich die Figuren zu der Zeit, die die Ebene des Diskurses übersteigt und auch thematisch aufgegriffen wird. Der Ich-Erzähler im Kurzen Brief kommt zu der Einsicht, dass inn die Verfolgung der physischen Zeit an der nötigen Aufmerksamkeit gehindert hat. Er träumt von einer anderen Zeit und stellt im Laufe der Geschichte fest, dass die andere Zeit im Innern seiner selbst zu suchen ist. Eine andere Zeit bedeutet für die Figuren innezuhalten, aufmerksam zu werden, in dem gegenwärtigen Augenblick zu existieren und durch diesen neu gewonnenen Zustand zu authentischen Einsichten zu gelangen.

Auch in weiteren Erzählungen ist die physische Zeit negativ konnotiert, sie schrumpft durch qualitative augenblickhafte Einsichten zum Stillstand. Sorger verwirft beispielsweise die chronologische Zeit. Loser, die Hauptfigur in Der Chinese des Schmerzes, schöpft die wesentlichen Erkenntnisse durch eine produktive Auseinandersetzung mit der Vergangenheit, die in einem Zeitstillstand die Verschmelzung der Zeitebenen erreicht. Kobal, der Protagonist der Wiederholung, lebt auch in einer qualitativen Zeitebene, in einer tätigen Erinnerung, die von der physischen Zeit abgehoben ist.

Die thematisch behandelte Zeit reflektiert sich ferner auf ihre Gestaltung im Diskurs. Im Kurzen Brief und der Stunde der wahren Empfindung ist eine extreme Dehnung der Zeit die natürliche Konsequenz der durchgehenden Behandlung von einzelnen Momenten, die Handke als neugewonnene Erzählstrategie in den 1970er-Jahren auch in weiteren Werken beibehält, so in Langsame Heimkehr, Die Wiederholung, Mein Jahr in der Niemandsbucht, usf.

Ferner hat Der kurze Brief das Verhältnis zwischen Erinnerung und Reflexion in Handkes Poetik apostrophiert. Im Kurzen Brief wird die Geschichte durch die Verarbeitung der Vergangenheit präsentiert, die den Wechselbezug zwischen Gegenwart und Vergangenheit als Erkenntnis- und Schreibgrundlage verankert.

Die Zukunft wird demzufolge durch die Erinnerung determiniert. Die Gegenwart als Ort aufmerksamer Reflexionen führt zu epiphanischen Einsichten, in denen Vergangenheit, Gegenwart und Zukunft ineinander verschmelzen.

Die Norm des Textes wird im Kurzen Brief, aber auch in Handkes weiteren Werken durch den Welthorizont der Hauptfigur bestimmt. Trotz des Vorgangs, dass der Erzähler die Wandlungen der Hauptfigur bloß registriert, sich also neutral gegenüber diesen Veränderungen verhält, da er sie an keiner Stelle aus einem 
olympischen Blickwinkel kommentiert oder gar beurteilt, wird der Eindruck erweckt, dass die Hauptfigur in ihren Überlegungen und ihrem Verhalten gegenüber der Umgebung immer Recht behält. Sie bestimmt die Norm des Textes im Sinne von Werten, die implizit aus dem Text herauszulesen sind. Dieser Eindruck ergibt sich aus dem Fokus, der bei der Wandlung der Hauptfigur konsequent beibehalten wird. Da die Zustände nicht nur kontinuierlich, sondern auch ausführlich verfolgt werden, mit einem Reichtum an Einzelheiten, erweckt diese Strategie den Eindruck von Authentizität und schließlich Glaubwürdigkeit. Die Gestaltung der Hauptfigur, so wie sie im Kurzen Brief erzählstrategisch angewandt ist, folgt auch in weiteren Erzählungen einem Modell, in dem sie als einmalige Individualität dargestellt wird. Durch durchgehende Beleuchtung der Zustände der Hauptfigur wird sie in den Mittelpunkt der diegetischen Welt gestellt, wobei die restlichen Figuren nur so viel Raum durch ihre Äußerungen und ihr Verhalten beanspruchen, um die Überlegungen der Hauptfigur zu bestätigen. Sie fungieren als Spiegel, der die inneren Vorgänge der Hauptfigur bestätigt und rechtfertigt. Dies kommt in der Erzählung Der kurze Brief zum langen Abschied zum Vorschein, aber auch in Die Stunde der wahren Empfindung, Langsame Heimkehr, Kindergeschichte, Mein Jahr in der Niemandsbucht, usf. Durch den Diskurs wird somit erreicht, dass der Erzähler die Zustände der Hauptfigur nur registriert, sie also nicht beurteilt, und dabei doch die Weltsicht der Hauptfigur als Norm des Textes vermittelt.

Schließlich verankert der Kurze Brief die Stellung der metanarrativen Schicht in Handkes Poetik. Sie spiegelt sich in der programmatischen Positionierung wider, den privaten authentischen Geschichten den Eingang in ein literarisches Werk zu ermöglichen. Der kurze Brief ist durch Überlegungen durchströmt, welche Schreibmethoden für diese poetologische Position passend wären. So wird die persönliche Entwicklung als Hauptthema zum Anlass für eine metanarrative Auseinandersetzung mit dem Prozess des Schreibens. Die metanarrative Ebene als Thematisierung der Schreibtätigkeit ist in diesem Kontext mit den intertextuellen Bezügen eng verknüpft.

Die enge Verknüpfung zwischen Leben und Schreiben, zwischen Authentizität und Fiktionalisierung offenbart sich im Kurzen Brief ferner in der Positionierung der Hauptfigur als Schriftsteller, der eine existenzielle Krise durchlebt. Der Schriftsteller ist die Instanz, die durch seine Schreibtätigkeit das Private als Vergängliche in einer Dauer bewahrt. Dieses große Geheimnis, das in vielen Werken immer wieder variiert und als persönliche Erlösung des Schriftstellers unterstrichen wird, wird im Kurzen Brief zum langen Abschied zu Ende der Geschichte 
von Judith festgehalten, die verkündet, dass eine authentische Geschichte nun in einem literarischen Werk aufbewahrt ist.

\section{Literaturverzeichnis}

Batt, Kurt. „Leben in Zitat. Notizen zu Peter Handke“. Sinn und Form 1.6. Heft, 26.Jahr (1974): 603-623.

Brüggemann, Aminia Chronotopos Amerika bei Max Frisch, Peter Handke, Günter Kunert und Martin Walser. New York: Peter Lang, 1996.

Durzak, Manfred. Peter Handke und die deutsche Gegenwartsliteratur. Stuttgart: Kohlhammer, 1982.

Elm, Theo. „Die Fiktion eines Entwicklungsromans. Zur Erzählstrategie in Peter Handkes Roman 'Der kurze Brief zum langen Abschied'." $\mathrm{Zu}$ Peter Handke. Zwischen Tradition und Experiment. Hg. Norbert Honsza. Stuttgart: Ernst Klett, 1983. 55-77.

Handke, Peter. Der kurze Brief zum langen Abschied. Frankfurt am Main: Suhrkamp, 2001.

Herwig, Malte. Meister der Dämmerung. Peter Handke. Eine Biographie. München: Deutsche Verlags-Anstalt, 2010.

Höller, Hans. Eine ungewöhnliche Klassik nach 1945. Das Werk Peter Handkes. Frankfurt am Main: Suhrkamp, 2013.

Király, Edit. „On the road with Peter Handke. Amerikanische (T)räume in der Erzählung Der kurze Brief zum langen Abschied." Das Wort sei gewagt. (Hg): Attila Bombitz und Katharina Pektor. Wien: Praesens Verlag, 2019. 89-99.

Krajenbrink, Marieke. Intertextualität als Konstruktionsprinzip. Amsterdam: Rodopi, 1996.

Krstanović, Andjelka. „Authentizität und Fiktionalisierung. Narrative Überlegungen zu Peter Handkes Erzählung 'Wunschloses Unglück'." Sprachkunst. Beiträge zur Literaturwissenschaft 2019/2 (2020), 231-254.

Krstanović, Andjelka. „Die Erzählverfahren in Die linkshändige Frau“ (in Druck). Facta Universitatis, Series: Linguistic and Literature Vol. 18, No 1 (2020).

Mixner, Manfred. Peter Handke. Kronberg: Athenäum Verlag, 1977.

Neis, Edgar. Peter Handke. Die Angst des Tormanns beim Elfmeter. Der kurze Brief zum langen Abschied. Wunschloses Unglück. Königs Erläuterungen und Materialien, Hollfeld/Ofr: Bange Verlag, 1978.

Renner, Rolf Günter. Peter Handke. Stuttgart: Metzler, 1985.

Schmidt, Volker. Die Entwicklung der Sprachkritik im Werk von Peter Handke und Elfriede Jelinek. Diss., Heidelberg, 2008. 
Winkelmann, Christine. Wahrnehmung und Weltbezug bei Botho Strauss und Peter Handke. Frankfurt am Main: Peter Lang, 1990.

\section{НАРАТИВНИ ЗАОКРЕТ У ХАНДКЕОВОМ КРАТКОМ ПИСМУ ЗА ДУГИ PACTAHAK}

Приповијетка Петера Хандкеа Кратко писмо за дуги растанак заједно са приповијетком Безжељна несрећа, настала такође 1971. године, показује значајан отклон у односу на наративне стратегије из 60-их година. Стога стратегије приповиједања у поменутим дјелима представљају заокрет у ауторовом књижевном опусу, те су као основне конституенте Хаднкеове поетике задржане и у каснијим дјелима. У раду су анализиране наративне технике у Кратком писму за дуги растанак, те позиција предметне приповијетке у односу на укупан опус аутора.

Кључне ријечи: Петер Хандке, приповијетка, приповиједне стратегије, наративни заокрет, поетика. 